\title{
Radial Limits of Nonparametric PMC Surfaces with Intermediate Boundary Curvature
}

\author{
Mozhgan Nora Entekhabi and Kirk Eugene Lancaster*
}

\begin{abstract}
The influence of the geometry of the domain on the behavior of generalized solutions of Dirichlet problems for elliptic partial differential equations has been an important subject for over a century. We investigate the boundary behavior of variational solutions $f$ of Dirichlet problems for prescribed mean curvature equations in a domain $\Omega \subset \mathbb{R}^{2}$ near a point $\mathcal{O} \in \partial \Omega$ under different assumptions about the curvature of $\partial \Omega$ on each side of $\mathcal{O}$. We prove that the radial limits at $\mathcal{O}$ of $f$ exist under different assumptions about the Dirichlet boundary data $\phi$, depending on the curvature properties of $\partial \Omega$ near $\mathcal{O}$.
\end{abstract}

\section{Introduction}

Let $\Omega$ be a bounded, locally Lipschitz domain in $\mathbb{R}^{2}$ and define $N f=\nabla \cdot T f=\operatorname{div}(T f)$, where $f \in C^{2}(\Omega)$ and $T f=\frac{\nabla f}{\sqrt{1+|\nabla f|^{2}}}$. Let $H \in C^{1, \lambda}(\bar{\Omega})$ for some $\lambda \in(0,1)$ and satisfy the condition

$$
\left|\int_{\Omega} H \eta d x\right| \leq \frac{1}{2} \int_{\Omega}|D \eta| d x \quad \text { for all } \eta \in C_{0}^{1}(\Omega)
$$

(e.g., see [14, (16.60)] and 15]). We wish to study the following

Dirichlet problem. Let $\phi \in L^{\infty}(\partial \Omega)$. Find a function $f \in C^{2}(\Omega) \cap C^{0}(\bar{\Omega})$ which satisfies

$$
\begin{array}{cc}
N f=2 H & \text { in } \Omega, \\
f=\phi & \text { on } \partial \Omega .
\end{array}
$$

If $\phi \in C^{0}(\partial \Omega)$ and a function $f \in C^{2}(\Omega) \cap C^{0}(\bar{\Omega})$ exists which satisfies $1.1-1.2$, this is a classical solution and, in this case, every appropriate approximate solution (e.g., Perron solutions, variational solution, viscosity solutions) will equal this classical solution. The geometry of $\Omega$ plays a critical role with regard to the existence of classical solutions when $\phi \in C^{0}(\partial \Omega)$. For some choices of domain $\Omega$ and boundary data $\phi$, no classical

Received July 23, 2020; Accepted November 12, 2020.

Communicated by Ulrich Menne.

2020 Mathematics Subject Classification. Primary: 35J67; Secondary: 35J93, 53A10.

Key words and phrases. prescribed mean curvature, Dirichlet problem, radial limits.

This research partially supported by NSF Award HRD-1824267.

*Corresponding author. 
solution of (1.1)-(1.2) exists; when $H \equiv 0$, much of the history (up to 1985) of this topic can be found in Nitsche's book [24] (e.g., §285, 403-418) and, for general $H$, one might consult [26]. (Appropriate "smallness of $\phi$ " conditions can imply the existence of classical solutions when $\Omega$ is not convex in the $H \equiv 0$ case (e.g., [24, §285\& \&412] and $[17,25,27,28]$ ) or when $\partial \Omega$ does not satisfy appropriate curvature conditions in the general case (e.g., [1, 16, 22]); however see [24, §411]. In [2], Bourni assumes $\partial \Omega$ and $\phi$ are $\left(C^{1, \alpha}\right)$ smooth, ignores the geometry of $\Omega$ and characterizes the "graph" of a variational solution which may include portions of the boundary cylinder $\partial \Omega \times \mathbb{R}$; in comparison, we do not assume any regularity for our boundary data $\phi$ and focus on the closure in $\bar{\Omega} \times \mathbb{R}$ of the graph of $f$ over $\Omega$.)

We wish to investigate the effects of the geometry of $\Omega$ on the behavior of a variational solution $f$ of $1.1-1.2$ near a point $\mathcal{O} \in \partial \Omega$; $\partial \Omega$ might be smooth or have a corner at $\mathcal{O}$ or $\phi$ might be discontinuous at $\mathcal{O}$. For convenience, we assume $\mathcal{O}=(0,0)$. In many cases, the approximate solution is unique since if $f, g \in C^{2}(\Omega)$ both satisfy (1.1) and $f=g$ almost everywhere on $\partial \Omega$, then $f=g$ in $\Omega$ (e.g., 12 , Theorem 5.1]); see, for example, [7, 645-6]) for a discussion of when Perron and variational solutions exist.

Let $\alpha$ and $\beta, \alpha<\beta<\alpha+2 \pi$, be the angles which the tangent rays to $\partial \Omega$ at $\mathcal{O}$ make with the positive $x$-axis such that

$$
\{(r \cos \theta, r \sin \theta): 0<r<\epsilon(\theta), \alpha<\theta<\beta\} \subset \Omega \cap B_{\delta}(\mathcal{O})
$$

for some $\delta>0$ and some function $\epsilon(\cdot):(\alpha, \beta) \rightarrow(0, \delta)$, and $\beta-\alpha \in(0,2 \pi)$ is the size of the "corner" at $\mathcal{O}$ of $\partial \Omega$. Here and throughout this note, we adopt the sign convention that the curvature of $\partial \Omega$ is nonnegative when $\Omega$ is convex and we denote by $\Lambda(\mathbf{x})$ the curvature of $\partial \Omega$ at points $\mathbf{x} \in \partial \Omega$ at which $\partial \Omega$ is smooth. Our primary interest is in the existence and behavior of the radial limits at $\mathcal{O}$,

$$
R f(\theta)=\lim _{r \downarrow 0} f(r \cos \theta, r \sin \theta),
$$

of a solution $f \in C^{2}(\Omega)$ of $(1.1)$; the existence of radial limits when $H \equiv 0$ was established in [18 (see also $6,8,19$ ) and this was extended to general $H$ in [7] (see also [9, 10, 21]). When $\Gamma$ is a $C^{2, \lambda}$ open subset of $\partial \Omega$ for some $\lambda \in(0,1), \mathcal{O} \in \Gamma, H \equiv 0$ and $f$ is a variational solution of (1.1)-(1.2), the following is known:

(i) If $\Lambda(\mathcal{O})<0$, then $R f(\theta)$ exists for every $\theta \in[\alpha, \beta]$ no matter how badly discontinuous $\phi$ is at $\mathcal{O}$ (see [11, Theorem 1.1]);

(ii) If $\Lambda(\mathcal{O})>0$, then there exist $\phi \in L^{\infty}(\partial \Omega) \cap C^{\infty}(\partial \Omega \backslash\{\mathcal{O}\})$ such that $R f(\theta)$ does not exist for any $\theta \in(\alpha, \beta)$ (i.e., $[20]$ ); 
(iii) If $R f(\theta)$ exists for some $\theta_{0} \in[\alpha, \beta]$, then $R f(\theta)$ exists for every $\theta \in(\alpha, \beta)$ (i.e., 9 , Theorem 2]);

(iv) If $\Lambda(\mathcal{O})=0$, then the existence of $R f(\theta)$ is unknown (but see [11, Theorem 1.1]);

(v) If $\phi$ is continuous at $\mathcal{O}$ or has a jump discontinuity at $\mathcal{O}$ and $\Lambda(\mathbf{x}) \geq 0$ for all $\mathbf{x} \in \Gamma$ near $\mathcal{O}$, then $R f(\theta)$ exists for every $\theta \in[\alpha, \beta]$ (see [18, 19]).

The equivalent statements when $H \not \equiv 0$ are

(vi) If $\Lambda(\mathcal{O})<-2|H(\mathcal{O})|$, then $R f(\theta)$ exists for every $\theta \in[\alpha, \beta]$ no matter how badly discontinuous $\phi$ is at $\mathcal{O}$ (see [11, Theorem 1.1]);

(vii) If $\Lambda(\mathcal{O})>2|H(\mathcal{O})|$, then there exist $\phi \in L^{\infty}(\partial \Omega) \cap C^{\infty}(\partial \Omega \backslash\{\mathcal{O}\})$ such that $R f(\theta)$ does not exist for any $\theta \in(\alpha, \beta)$ (i.e., [21, Theorem 3]);

(viii) If $R f(\theta)$ exists for some $\theta_{0} \in[\alpha, \beta]$, then $R f(\theta)$ exists for every $\theta \in(\alpha, \beta)$ (i.e., 9 , Theorem 2]);

(ix) If $-2|H(\mathcal{O})| \leq \Lambda(\mathcal{O})<2|H(\mathcal{O})|$, then the existence of $R f(\theta)$ is unknown;

(x) If $\phi$ is continuous at $\mathcal{O}$ or has a jump discontinuity at $\mathcal{O}$ and $\Lambda(\mathbf{x}) \geq|H(\mathbf{x})|$ for all $\mathbf{x} \in \Gamma$ near $\mathcal{O}$, then $R f(\theta)$ exists for every $\theta \in[\alpha, \beta]$ (see [7, 19]).

Our goals here are to determine what happens in case (ix) when $\partial \Omega$ is smooth near $\mathcal{O}$ and to determine the effects of the value of the curvature $\Lambda(\mathbf{x})$ on each side of $\mathcal{O}$ when $\partial \Omega$ is not smooth at $\mathcal{O}$ (i.e., $\beta-\alpha \neq \pi$ ).

\section{Preliminaries and theorems}

Let us assume that $B_{\delta}(\mathcal{O}) \cap \partial \Omega \backslash\{\mathcal{O}\}$ consists of two components, $\partial^{-} \Omega$ and $\partial^{+} \Omega$, which are smooth (i.e., $C^{2, \lambda}$ for some $\left.\lambda \in(0,1)\right)$ curves, $\partial^{-} \Omega$ is tangent to the ray $\theta=\alpha$ at $\mathcal{O}$ and $\partial^{+} \Omega$ is tangent to the ray $\theta=\beta$ at $\mathcal{O}$; here $(r, \theta)$ represents polar coordinates about $\mathcal{O}$ and $B_{\delta}(\mathcal{O})=\left\{\mathbf{x} \in \mathbb{R}^{2}:|\mathbf{x}-\mathcal{O}|<\delta\right\}$. We assume $\partial^{-} \Omega$ is an (open) subset of a $C^{2, \lambda}$-curve $\Sigma^{-}$which contains $\mathcal{O}$ as an interior point and $\partial^{+} \Omega$ is an (open) subset of a $C^{2, \lambda}$-curve $\Sigma^{+}$ which contains $\mathcal{O}$ as an interior point; if $\beta-\alpha=\pi$, we assume $\Sigma^{-}=\Sigma^{+}$(see Figure 2.1).

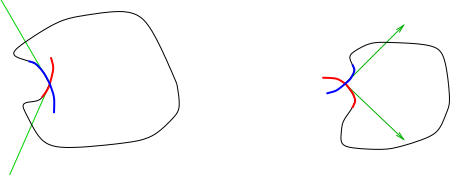

Figure 2.1: $\Sigma^{ \pm}$when $\beta-\alpha>\pi$ (left); $\Sigma^{ \pm}$when $\beta-\alpha<\pi$ (right). 
Let $f \in B V(\Omega) \cap C^{2}(\Omega)$ minimize the functional

$$
J(h)=\int_{\Omega} \sqrt{1+|D h|^{2}}+\int_{\Omega} 2 H h d \mathbf{x}+\int_{\partial \Omega}|u-\phi| d H_{1}
$$

for $h \in B V(\Omega)$, so that $f$ is the variational solution of 1.1$)-1.2$. (Our focus is local (near $\mathcal{O})$; if $\Omega$ was not bounded, we would consider $f \in C^{2}(\Omega)$ to be a generalized variational solution or just argue as in 21].) Let $R f(\theta)$ denote the radial limit of $f$ at $\mathcal{O}$ in the direction $\theta \in(\alpha, \beta)$,

$$
R f(\theta)=\lim _{r \downarrow 0} f(r \cos \theta, r \sin \theta),
$$

and set $R f(\alpha)=\lim _{\partial^{-} \Omega \ni \mathbf{x} \rightarrow \mathcal{O}} f^{*}(\mathbf{x})$ and $R f(\beta)=\lim _{\partial^{+} \Omega \ni \mathbf{x} \rightarrow \mathcal{O}} f^{*}(\mathbf{x})$ when these limits exist, where $f^{*}$ denotes the trace of $f$ on $\partial \Omega$. In [9] (together with [5]), the following two results were proven.

Proposition 2.1. (see [9, Theorem 1] and [5]) Let $f \in C^{2}(\Omega) \cap L^{\infty}(\Omega)$ satisfy (1.1] and suppose $\beta-\alpha>\pi$. Then for each $\theta \in(\alpha, \beta), R f(\theta)$ exists and $R f(\cdot)$ is a continuous function on $(\alpha, \beta)$ which behaves in one of the following ways:

(i) $R f$ is a constant function and all nontangential limits of $f$ at $\mathcal{O}$ exist.

(ii) There exist $\alpha_{1}, \alpha_{2} \in[\alpha, \beta]$ with $\alpha_{1}<\alpha_{2}$ such that

$$
R f(\theta) \text { is } \begin{cases}\text { constant } & \text { for } \alpha<\theta \leq \alpha_{1}, \\ \text { strictly monotonic } & \text { for } \alpha_{1} \leq \theta \leq \alpha_{2}, \\ \text { constant } & \text { for } \alpha_{2} \leq \theta<\beta .\end{cases}
$$

(iii) There exist $\alpha_{1}, \alpha_{2}$ and $\theta_{0}$ with $\alpha \leq \alpha_{1}<\theta_{0}<\theta_{0}+\pi<\alpha_{2} \leq \beta$ such that

$$
R f(\theta) \text { is } \begin{cases}\text { constant } & \text { for } \alpha<\theta \leq \alpha_{1}, \\ \text { strictly increasing (decreasing) } & \text { for } \alpha_{1} \leq \theta \leq \theta_{0}, \\ \text { constant } & \text { for } \theta_{0} \leq \theta \leq \theta_{0}+\pi, \\ \text { strictly decreasing (resp. increasing) } & \text { for } \theta_{0}+\pi \leq \theta \leq \alpha_{2}, \\ \text { constant } & \text { for } \alpha_{2} \leq \theta<\beta .\end{cases}
$$

Proposition 2.2. (see [9, Theorem 2] and [5]) Let $f \in C^{2}(\Omega) \cap L^{\infty}(\Omega)$ satisfy (1.1] and suppose $m=\lim _{\partial^{-} \Omega \ni \mathbf{x} \rightarrow \mathcal{O}} f(\mathbf{x})$ exists. Then for each $\theta \in(\alpha, \beta), R f(\theta)$ exists and $R f(\cdot)$ is a continuous function on $[\alpha, \beta)$, where $\operatorname{Rf}(\alpha):=m$. If $\beta-\alpha \leq \pi, R f$ can behave as in (i) or (ii) in Proposition 2.1. If $\beta-\alpha>\pi, R f$ can behave as in (i), (ii) or (iii) in Proposition 2.1. 
We shall prove

Theorem 2.3. Let $f$ be the variational solution of $(1.1)-(1.2)$. Suppose $\Gamma \subset \partial \Omega$ is a $C^{2, \lambda}$ (open) curve for some $\lambda \in(0,1), \mathcal{O} \in \Gamma, H$ is non-negative or non-positive in a neighborhood of $\mathcal{O}$ and $\Lambda(\mathcal{O})<2|H(\mathcal{O})|$. Then $R f(\theta)$ exists for each $\theta \in(\alpha, \beta), R f \in C^{0}((\alpha, \beta))$ and $R f$ behaves as in (i) or (ii) in Proposition 2.1. Further, if $\Lambda(\mathcal{O})<-2|H(\mathcal{O})|$, then $R f(\alpha)$ and $R f(\beta)$ both exist, $R f \in C^{0}([\alpha, \beta])$, and, in case (i) in Proposition 2.1, $f \in C^{0}(\Omega \cup\{\mathcal{O}\})$.

Example 2.4. Let $\Omega=\left\{(x, y) \in \mathbb{R}^{2}: x^{2}+y^{2}<1, x>0\right\}$ and set $H=1 / 2$ and $\phi(x, y)=\sin \left(\frac{\pi}{x^{2}+y^{2}}\right)$ for $(x, y) \neq \mathcal{O}=(0,0)$. Let $f \in C^{2}(\Omega)$ minimize 2.1 over $B V(\Omega)$. Then Theorem 2.3 implies that the radial limit $R f(\theta)$ exists for each $\theta \in(-\pi / 2, \pi / 2)$ even though $\phi$ has no limit at $\mathcal{O}$. The symmetry of the problem then implies that Proposition 2.1(ii) cannot hold and so the radial limits are all the same and $f$ has a nontangential limit at $\mathcal{O}$.

Theorem 2.5. Let $f \in L^{\infty}(\Omega) \cap C^{2}(\Omega)$ minimize (2.1) over $B V(\Omega)$ (i.e., $f$ is the variational solution of (1.1)-(1.2)). Suppose $H$ is non-negative or non-positive in a neighborhood of $\mathcal{O}$,

$$
\limsup _{\partial^{ \pm} \Omega \ni \mathbf{x} \rightarrow \mathcal{O}}(\Lambda(\mathbf{x})-2|H(\mathbf{x})|)<0
$$

and, if $\beta-\alpha<\pi, m=\lim _{\partial-\Omega \ni \mathbf{x} \rightarrow \mathcal{O}} f(\mathbf{x})$ exists. Then $R f(\theta)$ exists for each $\theta \in(\alpha, \beta)$ and $R f \in C^{0}(\alpha, \beta)$.

(a) If $\beta-\alpha \leq \pi$, Rf behaves as in (i) or (ii) in Proposition 2.1.

(b) If $\beta-\alpha>\pi, R f$ behaves as in (i), (ii) or (iii) in Proposition 2.1.

If, in addition, $\limsup _{\partial^{ \pm} \Omega \ni \mathbf{x} \rightarrow \mathcal{O}}(\Lambda(\mathbf{x})+2|H(\mathbf{x})|)<0$, then $R f(\alpha)$ and $R f(\beta)$ both exist, $R f \in C^{0}([\alpha, \beta])$, and, in case (i) in Proposition 2.1, $f \in C^{0}(\Omega \cup\{\mathcal{O}\})$.

As noted previously (e.g., [9]), the "gliding hump" construction (which depends on the existence of classical solutions of (1.1)-(1.2) ) cannot be successfully used when $\beta-\alpha>\pi$. When $\beta-\alpha<\pi$ and $(2.2)$ holds, local barriers for $(1.1)-(1.2)$ do not exist on $\partial^{ \pm} \Omega$ and the "gliding hump" construction in [20] and [21, Theorem 3] cannot be directly used in $\Omega$. One easily sees that this construction can be used to obtain a solution $g \in C^{2}\left(\Omega_{0}\right)$ of (1.1) such that none of the radial limits $R g(\theta)$ of $g$ at $\mathcal{O}$ exist whenever $\mathcal{O} \in \partial \Omega_{0}$ and $\Omega_{0}$ is a domain for which the Dirichlet problem has local barriers at each point of $\partial \Omega_{0}$; let us assume as in [21, Theorem 3] that $H=1 / 2$ and $\Omega_{0}$ is the disk of radius 1 centered at $(1,0)$. If we rotate $\Omega$ about $\mathcal{O}$ so $\Omega \cap B_{\delta}(\mathcal{O}) \subset \Omega_{0}$ and $\beta=-\alpha<\pi / 2$, define $\phi=g$ on $\partial\left(B_{\delta}(\mathcal{O}) \cap \Omega\right)$ and set $f=g$ in $\Omega \cap B_{\delta}(\mathcal{O})$, then $f \in C^{2}\left(\Omega \cap B_{\delta}(\mathcal{O})\right)$ satisfies 1.1), 
$\phi \in L^{\infty}\left(\partial\left(B_{\delta}(\mathcal{O}) \cap \Omega\right)\right) \cap C^{\infty}\left(\partial\left(B_{\delta}(\mathcal{O}) \cap \Omega\right) \backslash\{\mathcal{O}\}\right)$ and none of the radial limits $R f(\theta)$, $\alpha \leq \theta \leq \beta$, of $f$ at $\mathcal{O}$ exist (see Figure 2.2(a)). This shows the necessity of the assumption that $\lim _{\partial-\Omega \ni \mathbf{x} \rightarrow \mathcal{O}} f(\mathbf{x})$ exists when $\beta-\alpha<\pi$, although if $R f(\sigma)$ exists for any $\sigma \in(\alpha, \beta)$, we can split $\Omega$ into two pieces (see Figure 2.2(b)), apply [9, Theorem 2] twice and see that $R f(\theta)$ exists for all $\theta \in(\alpha, \beta)$ (which justifies (iii) \& (viii) in $\$ 1$ ).
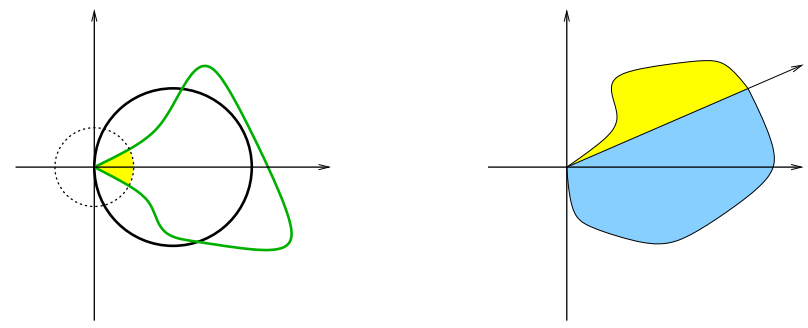

Figure 2.2: (a) $\Omega \cap B_{\delta}(\mathcal{O}) \subset \Omega_{0}$, (b) $R f(\sigma)$ exists, $\sigma>0$.

\section{Proofs}

Let $Q$ be the operator on $C^{2}(\Omega)$ given by

$$
Q f(\mathbf{x}):=N f(\mathbf{x})-2 H(\mathbf{x}), \quad \mathbf{x} \in \Omega .
$$

Let $\nu$ be the exterior unit normal to $\partial \Omega$, defined almost everywhere on $\partial \Omega$. At every point $\mathbf{y} \in \partial \Omega$ for which $\partial \Omega$ is a $C^{1}$ curve in a neighborhood of $\mathbf{y}, \widehat{\nu}$ denotes a continuous extension of $\nu$ to a neighborhood of $\mathbf{y}$. Finally we adopt the convention used in [3, p. 178] with regard to the meaning of phrases like " $T \psi(\mathbf{y}) \cdot \nu(\mathbf{y})=1$ at a point $\mathbf{y} \in \partial \Omega$ " and the notation, definitions and conventions used in [11], including upper and lower Bernstein pairs $\left(U^{ \pm}, \psi^{ \pm}\right)$, which we quote below.

Definition 3.1. Given a locally Lipschitz domain $\Omega$, an upper Bernstein pair $\left(U^{+}, \psi^{+}\right)$ for a curve $\Gamma \subset \partial \Omega$ and a function $H$ in (3.1) is a domain $U^{+}$and a function $\psi^{+} \in$ $C^{2}\left(U^{+}\right) \cap C^{0}\left(\overline{U^{+}}\right)$such that $\Gamma \subset \partial U^{+}, \nu$ is the exterior unit normal to $\partial U^{+}$at each point of $\Gamma$ (i.e., $U^{+}$and $\Omega$ lie on the same side of $\Gamma$ ), $Q \psi^{+} \leq 0$ in $U^{+}$, and $T \psi^{+} \cdot \nu=1$ almost everywhere on $\Gamma$ in the same sense as in $[3]$; that is, for almost every $\mathbf{y} \in \Gamma$,

$$
\lim _{U^{+} \ni \mathbf{x} \rightarrow \mathbf{y}} \frac{\nabla \psi^{+}(\mathbf{x}) \cdot \widehat{\nu}(\mathbf{x})}{\sqrt{1+\left|\nabla \psi^{+}(\mathbf{x})\right|^{2}}}=1 .
$$

Definition 3.2. Given a domain $\Omega$ as above, a lower Bernstein pair $\left(U^{-}, \psi^{-}\right)$for a curve $\Gamma \subset \partial \Omega$ and a function $H$ in $(3.1)$ is a domain $U^{-}$and a function $\psi^{-} \in C^{2}\left(U^{-}\right) \cap C^{0}\left(\overline{U^{-}}\right)$ such that $\Gamma \subset \partial U^{-}, \nu$ is the exterior unit normal to $\partial U^{-}$at each point of $\Gamma$ (i.e., $U^{-}$and $\Omega$ lie on the same side of $\Gamma), Q \psi^{-} \geq 0$ in $U^{-}$, and $T \psi^{-} \cdot \nu=-1$ almost everywhere on $\Gamma$ (in the same sense as above). 
The argument which establishes [14, Corollary 14.13], together with boundary regularity results (e.g., [2,23]), are noted in [11, Remark 1] and imply the following

Lemma 3.3. Suppose $\Delta$ is a $C^{2, \lambda}$ domain in $\mathbb{R}^{2}$ for some $\lambda \in(0,1), \mathbf{y} \in \partial \Omega$ and $\Lambda(\mathbf{y})<2|H(\mathbf{y})|$, where $\Lambda(\mathbf{y})$ denotes the curvature of $\partial \Delta$ at $\mathbf{y}$. If $H$ is non-negative in $U \cap \Omega$ for some neighborhood $U$ of $\mathbf{y}$, then there exist $\tau>0$ and an upper Bernstein pair $\left(U^{+}, \psi^{+}\right)$for $(\Gamma, H)$, where $\Gamma=B_{\tau}(\mathbf{y}) \cap \partial \Omega$ and $U^{+}=B_{\tau}(\mathbf{y}) \cap \Omega$. If $H$ is non-positive in $U \cap \Omega$ for some neighborhood $U$ of $\mathbf{y}$, then there exist $\tau>0$ and a lower Bernstein pair $\left(U^{-}, \psi^{-}\right)$for $(\Gamma, H)$, where $\Gamma=B_{\tau}(\mathbf{y}) \cap \partial \Omega$ and $U^{-}=B_{\tau}(\mathbf{y}) \cap \Omega$.

Proof of Theorem 2.3. The claims in the last sentence of the theorem follow from 11 , Theorem 1.1]. Since the remainder of the conclusion of the theorem concerns interior radial limits, we may assume that $f \in C^{0}(\bar{\Omega} \backslash\{\mathcal{O}\})$ (i.e., $f \in C^{2}(\Omega)$ and, if necessary, we could replace $\Omega$ by a set $U \subset \Omega$ such that $\partial U \cap \partial \Omega=\{\mathcal{O}\}, \partial U$ has the same tangent rays at $\mathcal{O}$ as does $\partial \Omega$ and the curvature $\Lambda^{*}$ of $\partial U$ satisfies $\left.\Lambda^{*}(\mathcal{O})<2|H(\mathcal{O})|\right)$.

Let $z_{1}=\liminf _{\Omega \ni \mathbf{x} \rightarrow \mathcal{O}} f(\mathbf{x})$ and $z_{2}=\lim \sup _{\Omega \ni \mathbf{x} \rightarrow \mathcal{O}} f(\mathbf{x})$; if $z_{1}=z_{2}$, then Proposition 2.1(i) holds and thus we assume $z_{1}<z_{2}$. Set $S_{0}=\{(\mathbf{x}, f(\mathbf{x})): \mathbf{x} \in \Omega\}$. Since $f$ minimizes $J$ in (2.1), we see that the area of $S_{0}$ is finite; let $M_{0}$ denote this area. For $\delta \in(0,1)$, set

$$
p(\delta)=\sqrt{\frac{8 \pi M_{0}}{\ln \left(\frac{1}{\delta}\right)}} .
$$

Let $E=\left\{(u, v): u^{2}+v^{2}<1\right\}$. As in [6,21], there is a parametric description of the surface $S_{0}$,

$$
Y(u, v)=(a(u, v), b(u, v), c(u, v)) \in C^{2}\left(E: \mathbb{R}^{3}\right),
$$

which has the following properties:

(a) $Y$ is a diffeomorphism of $E$ onto $S_{0}$.

$\left(\mathrm{a}_{2}\right)$ Set $G(u, v)=(a(u, v), b(u, v)),(u, v) \in E$. Then $G \in C^{0}\left(\bar{E}: \mathbb{R}^{2}\right)$.

$\left(\mathrm{a}_{3}\right)$ Set $\sigma(\mathcal{O})=G^{-1}(\partial \Omega \backslash\{\mathcal{O}\})$; then $\sigma(\mathcal{O})$ is a connected (open) arc of $\partial E$ and $G$ maps $\sigma(\mathcal{O})$ onto $\partial \Omega \backslash\{\mathcal{O}\}$. We may assume the endpoints of $\sigma(\mathcal{O})$ are $\mathbf{o}_{1}$ and $\mathbf{o}_{2}$. (Note that $\mathbf{o}_{1}$ and $\mathbf{o}_{2}$ are not assumed to be distinct.)

(a 4$) Y$ is conformal on $E: Y_{u} \cdot Y_{v}=0, Y_{u} \cdot Y_{u}=Y_{v} \cdot Y_{v}$ on $E$.

(as) $\triangle Y:=Y_{u u}+Y_{v v}=2 H(Y) Y_{u} \times Y_{v}$ on $E$.

Let $\zeta(\mathcal{O})=\partial E \backslash \sigma(\mathcal{O})$; then $G(\zeta(\mathcal{O}))=\{\mathcal{O}\}$ and $\mathbf{o}_{1}$ and $\mathbf{o}_{2}$ are the endpoints of $\zeta(\mathcal{O})$.

Suppose first that $\mathbf{o}_{1} \neq \mathbf{o}_{2}$. From the Courant-Lebesgue Lemma (e.g., Lemma 3.1 in $[4]$, we see that there exists $\rho=\rho(\delta, \mathbf{w}) \in(\delta, \sqrt{\delta})$ such that the arc length $l_{\rho}=l_{\rho(\delta, \mathbf{w})}$ 
of $Y\left(C_{\rho(\delta, \mathbf{w})}(\mathbf{w})\right)$ is less than $p(\delta)$, for each $\delta \in(0,1)$ and $\mathbf{w} \in \partial E$; here $C_{r}(\mathbf{w})=\{(u, v) \in$ $E:|(u, v)-\mathbf{w}|=r\}$. Set $E_{r}(\mathbf{w})=\{(u, v) \in E:|(u, v)-\mathbf{w}|<r\}, E_{r}^{\prime}(\mathbf{w})=G\left(E_{r}(\mathbf{w})\right)$ and $C_{r}^{\prime}(\mathbf{w})=G\left(C_{r}(\mathbf{w})\right)$. Choose $\delta_{1}>0$ such that $2 \sqrt{\delta_{1}}<\left|\mathbf{o}_{1}-\mathbf{o}_{2}\right|$. Let $\mathbf{w}_{0} \in \zeta(\mathcal{O})$ be the "midpoint" of $\mathbf{o}_{1}$ and $\mathbf{o}_{2}$, so that $\sqrt{\delta_{1}}<\left|\mathbf{w}_{0}-\mathbf{o}_{1}\right|=\left|\mathbf{w}_{0}-\mathbf{o}_{2}\right|$. Set $\mathcal{C}=C_{\rho\left(\delta_{1}, \mathbf{w}_{0}\right)}^{\prime}\left(\mathbf{w}_{0}\right)$; then $\{(\mathbf{x}, f(\mathbf{x})): \mathbf{x} \in \mathcal{C}\}\left(=Y\left(C_{\rho\left(\delta_{1}, \mathbf{w}_{0}\right)}\left(\mathbf{w}_{0}\right)\right)\right)$ is a curve of finite length $l_{\rho\left(\delta_{1}, \mathbf{w}_{0}\right)}$ with endpoints $\left(\mathcal{O}, z_{a}\right)$ and $\left(\mathcal{O}, z_{b}\right)$ for some $z_{a}, z_{b} \in \mathbb{R}$. Notice, in particular, that the graph of $f$ over $\mathcal{C}$ is either continuous at $\mathcal{O}$ (if $z_{a}=z_{b}$ ) or has a jump discontinuity at $\mathcal{O}$ (if $z_{a} \neq z_{b}$ ).

We may now argue as in 19 . Let $\Omega_{0}=G\left(E_{\rho\left(\delta_{1}, \mathbf{w}_{0}\right)}\left(\mathbf{w}_{0}\right)\right)=E_{\rho\left(\delta_{1}, \mathbf{w}_{0}\right)}^{\prime}\left(\mathbf{w}_{0}\right)$, so that $\partial \Omega_{0}=\mathcal{C} \cup\{\mathcal{O}\}$. From the Courant-Lebesgue Lemma and the general comparison principle (see [12, Theorem 5.1]), we see that $Y$ is uniformly continuous on $E_{\rho\left(\delta_{1}, \mathbf{w}_{0}\right)}\left(\mathbf{w}_{0}\right)$ and so extends to a continuous function on the closure of $E_{\rho\left(\delta_{1}, \mathbf{w}_{0}\right)}\left(\mathbf{w}_{0}\right)$. From Steps 2, 4 and 5 of [21] and with [5] replacing Step 3 of [21], we see that there exist $\alpha_{0}, \beta_{0} \in[\alpha, \beta]$ with $\alpha_{0}<\beta_{0}$ such that

$$
\left\{r(\cos \theta, \sin \theta): 0<r<\epsilon_{0}(\theta), \alpha_{0}<\theta<\beta_{0}\right\} \subset \Omega_{0} \cap B_{\delta_{0}}(\mathcal{O})
$$

for some function $\epsilon_{0}(\cdot):(\alpha, \beta) \rightarrow\left(0, \delta_{0}\right)$ and the radial limits $R f(\theta)$ of $f$ at $\mathcal{O}$ exist for $\alpha_{0} \leq \theta \leq \beta_{0}$. Since $\partial \Omega$ is $\left(C^{2, \lambda}\right)$ smooth near $\mathcal{O}$, we have $\beta-\alpha=\pi$ and so $\beta_{0}-\alpha_{0} \leq \pi$. (We note that $z_{a}=z_{b}$ when $\mathbf{o}_{1} \neq \mathbf{o}_{2}$ and $\beta_{0}-\alpha_{0} \leq \pi$ implies $f \in C^{0}(\bar{\Omega})$, a contradiction, and so $z_{a} \neq z_{b}$.) The existence of $R f(\cdot)$ on $(\alpha, \beta)$ now follows from two applications of [9, Theorem 2], one in the domain $\left\{(r \cos \theta, r \sin \theta) \in \Omega: r>0,\left(\alpha_{0}+\beta_{0}\right) / 2<\theta<\beta\right\}$ and one in the domain $\left\{(r \cos \theta, r \sin \theta) \in \Omega: r>0, \alpha<\theta<\left(\alpha_{0}+\beta_{0}\right) / 2\right\}$.

Suppose second that $\mathbf{o}=\mathbf{o}_{1}=\mathbf{o}_{2}$ and $\zeta(\mathcal{O})=\{\mathbf{o}\}$. Let us assume that $H$ is non-negative in a neighborhood of $\mathcal{O}$; here $H(Y(u, v))$ means $H(a(u, v), b(u, v))$. From Lemma 3.3 , we see that an upper Bernstein pair $\left(U^{+}, \psi^{+}\right)$for $\left(\Gamma_{1}, H\right)$ exists, where $U^{+}=\Omega \cap B_{\tau}(\mathcal{O})$ and $\Gamma_{1}=\Gamma \cap B_{\tau}(\mathcal{O})$ for some $\tau>0$; let $q$ denote a modulus of continuity for $\psi^{+}$. Then $T \psi^{+} \cdot \nu=+1$ (in the sense of $[3]$ ) on $\Gamma_{1}$ and, for each $C \in \mathbb{R}$, $Q\left(\psi^{+}+C\right)=Q\left(\psi^{+}\right) \leq 0$ on $\Omega \cap U^{+}$or equivalently

$$
N\left(\psi^{+}+C\right)(\mathbf{x}) \leq 2 H(\mathbf{x})=N f(\mathbf{x}) \quad \text { for } \mathbf{x} \in \Omega \cap U^{+} .
$$

From the Courant-Lebesgue Lemma, we see that there exists $\rho=\rho(\delta, \mathbf{w}) \in(\delta, \sqrt{\delta})$ such that the arc length $l_{\rho}=l_{\rho(\delta, \mathbf{w})}$ of $Y\left(C_{\rho(\delta, \mathbf{w})}(\mathbf{w})\right)$ is less than $p(\delta)$, for each $\delta \in(0,1)$ and $\mathbf{w} \in \partial E$.

Let us assume that $\delta \in(0,1)$ is small enough that $p(\delta)<\tau$, so that $G(\mathbf{w}) \in U^{+}$ for each $\mathbf{w} \in E$ with $|\mathbf{w}-\mathbf{o}| \leq \sqrt{\delta}$ and $G(\mathbf{w}) \in \Gamma_{1}$ for each $\mathbf{w} \in \partial E$ with $|\mathbf{w}-\mathbf{o}| \leq$ $\sqrt{\delta}$. Now $\psi^{+}-\psi^{+}(\mathbf{x}) \leq q(p(\delta))$ in $E_{\rho(\delta, \mathbf{o})}^{\prime}(\mathbf{o})$ for any $\mathbf{x} \in E_{\rho(\delta, \mathbf{o})}^{\prime}(\mathbf{o})$ and using (3.2) in conjunction with Finn's general comparison principle (see [12, Theorem 5.1]) implies that if $U \subset E_{\rho(\delta, \mathbf{o})}^{\prime}(\mathbf{o})$ is an open set, then

$$
f \leq \sup _{\Omega \cap \partial U} f+\psi^{+}-\inf _{\Omega \cap \partial U} \psi^{+} \leq \sup _{\Omega \cap \partial U} f+q(p(\delta)) \quad \text { in } U .
$$


Set

$$
k(\delta)=\inf _{\mathbf{u} \in C_{\rho(\delta, \mathbf{o})}(\mathbf{o})} c(\mathbf{u})=\inf _{\mathbf{x} \in C_{\rho(\delta, \mathbf{o})}^{\prime}(\mathbf{o})} f(\mathbf{x}) .
$$

Now $f \leq k(\delta)+p(\delta)$ on $C_{\rho(\delta, \mathbf{o})}^{\prime}(\mathbf{o})$ and $\psi^{+}-\inf _{C_{\rho(\delta, \mathbf{o})}^{\prime}(\mathbf{o})} \psi^{+} \leq q(p(\delta))$ in $E_{\rho(\delta, \mathbf{o})}^{\prime}(\mathbf{o})$ and so (3.3) implies

$$
f \leq k(\delta)+p(\delta)+\psi^{+}{ }_{C_{\rho(\delta, \mathbf{o})}^{\prime}(\mathbf{o})} \psi^{+} \leq k(\delta)+p(\delta)+q(p(\delta))
$$

or

$$
\sup _{E_{\rho(\delta, \mathbf{o})}^{\prime}(\mathbf{o})} f \leq \inf _{C_{\rho(\delta, \mathbf{o})}^{\prime}(\mathbf{o})} f+p(\delta)+q(p(\delta))
$$

Since $\sup _{\left.E_{\rho(\delta, \mathbf{o})}^{\prime}(\mathbf{o})\right)} f \geq z_{2}$

$$
\inf _{C_{\rho(\delta, \mathbf{o})}^{\prime}(\mathbf{o})} f \geq z_{2}-p(\delta)-q(p(\delta))=z_{2}-o(\delta) \quad \text { for each } \delta>0 .
$$

Let $z(\delta)=z_{2}-2 p(\delta)-q(p(\delta))$ and

$$
M(\delta)=\left\{\mathbf{x} \in E_{\rho(\delta, \mathbf{o})}^{\prime}(\mathbf{o}): f(\mathbf{x})>z(\delta)\right\} .
$$

(Recall $f \in C^{0}(\bar{\Omega} \backslash\{\mathcal{O}\})$ and $c \in C^{0}(\bar{E} \backslash\{\mathbf{o}\})$.) Then for each $\delta \in\left(0, p^{-1}(\tau)\right)$, (3.4) implies $f \geq z_{2}-p(\delta)-q(p(\delta))>z(\delta)$ on $C_{\rho(\delta, \mathbf{o})}^{\prime}(\mathbf{o})$ and so

$$
C_{\rho(\delta, \mathbf{o})}^{\prime}(\mathbf{o}) \subset M(\delta) \quad \text { and } \quad \mathcal{O} \in \overline{M(\delta)}
$$

Let $V(\delta)$ denote the component of $M(\delta)$ which contains $C_{\rho(\delta, \mathbf{o})}^{\prime}(\mathbf{o})$. We claim that $\mathcal{O} \in$ $\overline{V(\delta)}$. Suppose otherwise; then there is a curve $\mathcal{I}$ in $E_{\rho(\delta, \mathbf{o})}^{\prime}(\mathbf{o})$ (with endpoints $\mathbf{x}^{-} \in \partial^{-} \Omega$ and $\mathbf{x}^{+} \in \partial^{+} \Omega$ ) such that $f \leq z(\delta)$ on $\mathcal{I}$. Let $\Omega(\mathcal{I})$ be the component of $\Omega \backslash \mathcal{I}$ whose closure contains $\mathcal{O}$. Then 3.3 implies that

$$
f \leq \sup _{\mathcal{I}} f+q(p(\delta)) \leq z(\delta)+q(p(\delta))=z_{2}-2 p(\delta) \quad \text { in } \Omega(\mathcal{I})
$$

and so $\limsup _{E \ni \mathbf{w} \rightarrow \mathbf{o}} c(\mathbf{w}) \leq z_{2}-2 p(\delta)<z_{2}$, which is a contradiction; hence no such curve $\mathcal{I}$ exists and $\mathcal{O} \in \overline{V(\delta)}$.

Now $f \geq z(\delta)$ in $V(\delta)$ for each $\delta \in\left(0, p^{-1}(\tau)\right)$. Let $\mathcal{C}$ be any curve in $\Omega$ which starts at a point $\mathbf{x}_{0} \in C_{\rho\left(p^{-1}(\tau), \mathbf{o}\right)}^{\prime}(\mathbf{o})$ and ends at $\mathcal{O}$ such that

$$
\mathcal{C} \subset V(\delta) \quad \text { for each } \delta \in\left(0, p^{-1}(\tau)\right) .
$$

Since $\lim \inf _{\mathcal{C} \ni \mathbf{x} \rightarrow \mathcal{O}} f(\mathbf{x}) \geq \lim _{\delta \downarrow 0} z(\delta)=z_{2}$ and $z_{2}=\limsup _{\Omega \ni \mathbf{x} \rightarrow \mathcal{O}} f(\mathbf{x})$, we see that

$$
\lim _{\mathcal{C} \ni \mathbf{x} \rightarrow \mathcal{O}} f(\mathbf{x})=z_{2}
$$


We may, if we wish, extend $\mathcal{C}$ by adding to $\mathcal{C}$ a curve from $\mathbf{x}_{0}$ to a point on $\partial \Omega \backslash$ $\overline{E_{\rho\left(p^{-1}(\tau), \mathbf{o}\right)}^{\prime}(\mathbf{o})}$.

Now we modify the argument in the proof of [9, Theorem 2] to show that $R f(\theta)=z_{2}$ for all $\theta \in(\alpha, \beta)$; that is, we shall show that the nontangential limit of $f$ at $\mathcal{O}$ exists and equals $z_{2}$. Let $\alpha^{\prime}, \beta^{\prime} \in(\alpha, \beta)$ with $\alpha^{\prime}<\beta^{\prime}$.
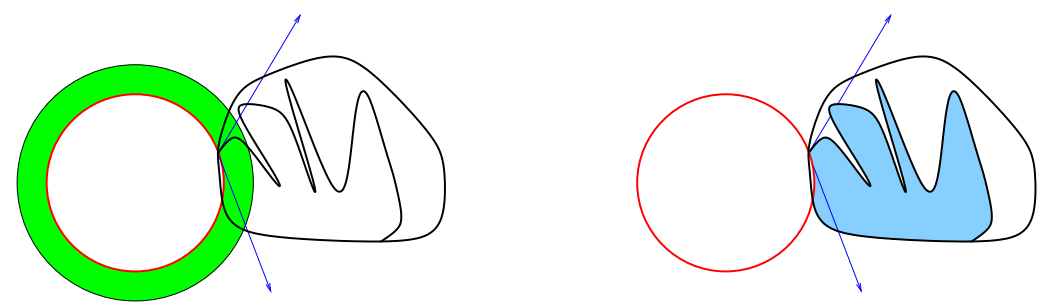

Figure 3.1: $\Omega, \mathcal{A}_{-}$and $\mathcal{C}$ (left); $\Omega_{2}$ (right).

Let $H_{0}=\sup _{B_{\delta_{0}}(\mathcal{O}) \cap \Omega} H$ and fix $c_{0} \in\left(-\frac{1}{4 c_{0} H_{0}}, 0\right)$. Set $r_{1}=\frac{1-\sqrt{1+4 c_{0} H_{0}}}{2 H_{0}}$ and $r_{2}=$ $\frac{1+\sqrt{1+4 c_{0} H_{0}}}{2 H_{0}}$ (see 21 , p. 171], 13]). Let $\mathcal{A}_{ \pm}$be annuli with inner boundaries $\partial_{1} \mathcal{A}_{ \pm}$with equal radii $r_{1}$ and outer boundaries $\partial_{2} \mathcal{A}_{ \pm}$with equal radii $r_{2}$ such that $\mathcal{O} \in \partial_{1} \mathcal{A}_{+} \cap \partial_{1} \mathcal{A}_{-}$, $\partial_{1} \mathcal{A}_{+}$is tangent to the ray $\theta=\beta^{\prime}$ at $\mathcal{O}, \partial_{1} \mathcal{A}_{-}$is tangent to the ray $\theta=\alpha^{\prime}$ at $\mathcal{O}$ and $\partial_{1} \mathcal{A}_{ \pm} \cap\left\{(r \cos \theta, r \sin \theta): 0<r<\delta_{0}, \alpha^{\prime}<\theta<\beta^{\prime}\right\}=\emptyset$ (see Figure 3.1). Let $h_{ \pm}=h\left(\widehat{r}_{ \pm}\right)$ denote unduloid surfaces defined respectively on $\mathcal{A}_{ \pm}$with constant mean curvature $-H_{0}$ which become vertical at $\widehat{r}_{ \pm}=r_{1}, r_{2}$ and make contact angles of $\pi$ and 0 with the vertical cylinders $\widehat{r}_{ \pm}=r_{2}$ and $\widehat{r}_{ \pm}=r_{1}$ respectively, where $\widehat{r}_{+}(\mathbf{x})=\left|\mathbf{x}-\mathbf{c}_{+}\right|, \widehat{r}_{-}(\mathbf{x})=\left|\mathbf{x}-\mathbf{c}_{-}\right|, \mathbf{c}_{+}$ denotes the center of the annulus $\mathcal{A}_{+}$and $\mathbf{c}_{-}$denotes the center of the annulus $\mathcal{A}_{-}$. With respect to the upward direction, the graphs of $h_{ \pm}$over $\mathcal{A}_{ \pm}$have constant mean curvature $-H_{0}$ and the graphs of $-h_{ \pm}$over $\mathcal{A}_{ \pm}$have constant mean curvature $H_{0}$.

Set $\tau_{1}=\min \left\{\tau, r_{2}-r_{1}\right\}$. Let $\delta \in\left(0, p^{-1}\left(\tau_{1}\right)\right)$. Since $\mathcal{C}$ is a curve in $\Omega$ with $\mathcal{O}$ as an endpoint, there exists $\mathbf{x}(\delta) \in \mathcal{C} \cap C_{\rho(\delta, \mathbf{o})}^{\prime}(\mathbf{o})$ such that the portion $\mathcal{C}(\delta)$ of $\mathcal{C}$ between $\mathcal{O}$ and $\mathbf{x}(\delta)$ lies in $E_{\rho(\delta, \mathbf{o})}^{\prime}(\mathbf{o})$ and divides $E_{\rho(\delta, \mathbf{o})}^{\prime}(\mathbf{o})$ into two components. Let $U_{+}$be the component of $E_{\rho(\delta, \mathbf{o})}^{\prime}(\mathbf{o}) \backslash \mathcal{C}(\delta)$ whose closure contains a portion of $\partial^{+} \Omega$ and $U_{-}$be the component of $E_{\rho(\delta, \mathbf{o})}^{\prime}(\mathbf{o}) \backslash \mathcal{C}(\delta)$ whose closure contains a portion of $\partial^{-} \Omega$ (see Figure 3.2 with $C_{\rho(\delta, \mathbf{o})}^{\prime}(\mathbf{o})$ (green) and $\mathcal{C}($ red $\left.)\right)$.
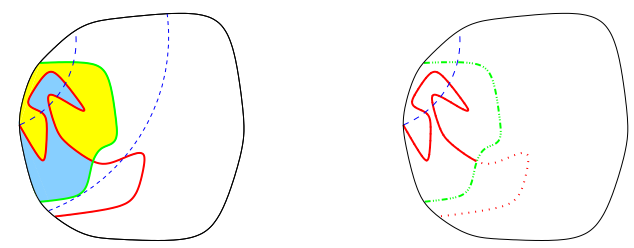

Figure 3.2: Left: $U_{+}$(yellow), $U_{-}$(blue); Right: $\mathcal{C}(\delta)$ (red). 
Since $\mathcal{C}(\delta) \subset V(\delta)$

$$
f(\mathbf{x}) \geq z(\delta) \quad \text { for } \mathbf{x} \in \mathcal{C}(\delta)
$$

and, in particular, $f(\mathbf{x}(\delta)) \geq z(\delta)$. Since $|f(\mathbf{x}(\delta))-f(\mathbf{y})| \leq l_{\rho(\delta, \mathbf{o})}<p(\delta)$ for $\mathbf{y} \in C_{\rho(\delta, \mathbf{o})}^{\prime}(\mathbf{o})$, we see that

$$
f \geq z(\delta)-p(\delta) \quad \text { on } C_{\rho(\delta, \mathbf{o})}^{\prime}(\mathbf{o}) \cup \mathcal{C}(\delta)
$$

Let $q_{2}$ denote a modulus of continuity of $-h\left(\widehat{r}_{+}\right)$. Then

$$
f \geq z(\delta)-p(\delta)-q_{2}(p(\delta)) \quad \text { in } U_{+} \backslash \overline{B_{r_{1}}\left(c_{+}\right)}
$$

Thus

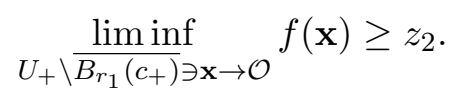

If we set $\Omega_{1}=U_{+} \backslash \overline{B_{r_{1}}\left(\mathbf{c}_{+}\right)}$and recall that $z_{2}=\lim \sup _{\Omega \ni \mathbf{x} \rightarrow \mathcal{O}} f(\mathbf{x})$, we have

$$
\lim _{\Omega_{1} \ni \mathbf{x} \rightarrow \mathcal{O}} f(\mathbf{x})=z_{2}
$$

(We note that $\Omega_{1}$ might not be connected (see Figure 3.3 ) and might even have an infinite number of components but one sees that this does not affect the comparison argument which establishes 3.5 .)

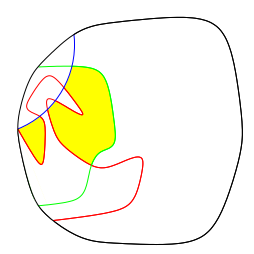

Figure 3.3: $\Omega$ and $\Omega_{1}$.

In a similar manner, we see that

$$
\lim _{\Omega_{2} \ni \mathbf{x} \rightarrow \mathcal{O}} f(\mathbf{x})=z_{2}
$$

where $\Omega_{2}=U_{-} \backslash \overline{B_{r_{1}}\left(\mathbf{c}_{-}\right)}$. Since $\Omega_{1} \cup \Omega_{2} \cup \mathcal{C}(\delta)=E_{\rho(\delta, \mathbf{o})}^{\prime}(\mathbf{o}) \backslash\left(\overline{B_{r_{1}}\left(\mathbf{c}_{+}\right) \cup B_{r_{1}}\left(\mathbf{c}_{-}\right)}\right)$, we see that $R f(\theta)=z_{2}$ for each $\theta \in\left(\alpha^{\prime}, \beta^{\prime}\right)$. Since $\alpha^{\prime}$ and $\beta^{\prime}$ are arbitrary (with $\alpha<\alpha^{\prime}<\beta^{\prime}<\beta$ ), Theorem 2.3 is proven.

Proof of Theorem 2.5. All of the claims in the theorem except those in the last sentence follow from [9, Theorem 1] and [5] (when $\beta-\alpha>\pi$ ) and [9, Theorem 2] and [5] (when $\beta-\alpha<\pi$ ). (When $\beta-\alpha=\pi$, all of the claims follow from Theorem 2.3 and [11.) 
The claims follow once we prove that the results of [11 hold under the assumptions of Theorem 2.5. Let us assume

$$
\limsup _{\partial^{ \pm} \Omega \ni \mathbf{x} \rightarrow \mathcal{O}}(\Lambda(\mathbf{x})+2|H(\mathbf{x})|)<0 .
$$

Suppose $\beta-\alpha>\pi$. Let $\delta_{1}>0$ be small enough that $B_{\delta_{1}}(\mathcal{O}) \cap \Omega \backslash \Sigma^{+}$has two components. Let $\Omega_{+}$be the component whose closure contains $B_{\delta_{1}}(\mathcal{O}) \cap \partial^{+} \Omega$ (see Figure 3.4 (left)) and notice that the tangent directions to $\partial \Omega_{+}$at $\mathcal{O}$ are $\alpha^{\prime}=\beta-\pi$ and $\beta$ and the curvature $\Lambda_{+}(\mathcal{O})$ of $\partial \Omega_{+}$at $\mathcal{O}$ satisfies

$$
\Lambda_{+}(\mathcal{O})<-2 H(\mathcal{O})
$$

since $\Lambda_{+}(\mathbf{x})=\Lambda(\mathbf{x})$ for $\mathbf{x} \in B_{\delta_{1}}(\mathcal{O}) \cap \partial^{+} \Omega$ and $(3.6)$ implies

$$
\Lambda_{+}(\mathcal{O})=\limsup _{\partial^{+} \Omega \ni \mathbf{x} \rightarrow \mathcal{O}} \Lambda_{+}(\mathbf{x})<-2|H(\mathcal{O})|
$$

By restricting $f$ to $\Omega_{+}$, we see that the existence of $R f(\beta)$ follows from [11]. A similar argument implies $R f(\alpha)$ also exists.
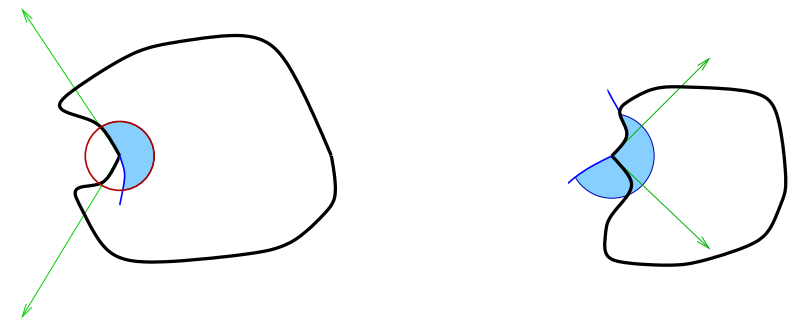

Figure 3.4: $\Omega_{+}$when $\beta-\alpha>\pi$ (left); $\Omega_{+}$when $\beta-\alpha<\pi$ (right).

Suppose $\beta-\alpha<\pi$. Then $R f(\alpha)$ exists and equals $m$. Let $\delta_{1}>0$ be small enough that $B_{\delta_{1}}(\mathcal{O}) \backslash \Sigma^{+}$has two components and let $\Omega_{+}$be the component which contains $B_{\delta_{1}}(\mathcal{O}) \cap \Omega$ (see Figure 3.4 (right)). Then the tangent directions to $\partial \Omega_{+}$at $\mathcal{O}$ are $\alpha^{\prime}=\beta-\pi$ and $\beta$ and, as before, the curvature $\Lambda_{+}(\mathcal{O})$ of $\partial \Omega_{+}$at $\mathcal{O}$ satisfies $\Lambda_{+}(\mathcal{O})<-2 H(\mathcal{O})$. Thus upper and lower Bernstein pairs $\left(U^{ \pm}, \psi^{ \pm}\right)$exist for $\Gamma=B_{\delta_{2}}(\mathcal{O}) \cap \partial \Omega_{+}$and $H$ when $\delta_{2} \in\left(0, \delta_{1}\right)$ is sufficiently small and $U^{ \pm}=B_{\delta_{2}}(\mathcal{O}) \cap \Omega_{+}$. We may parametrize $S_{1}=S_{0} \cap\left(B_{\delta_{2}}(\mathcal{O}) \times \mathbb{R}\right)$ in isothermal coordinates

$$
Y(u, v)=(a(u, v), b(u, v), c(u, v)) \in C^{2}\left(E: S_{1}\right)
$$

as in 11 with the properties noted there (e.g., $\left.a_{1}, \ldots, a_{5}\right)$ and prove in essentially the same manner as in [11] that $Y$ is uniformly continuous on $E$ and so extends to a continuous function on $\bar{E}$. The existence of $R f(\beta)$ then follows as in 11 . 


\section{Acknowledgments}

The authors would like to thank the referee for his/her efforts.

\section{References}

[1] M. Bergner, On the Dirichlet problem for the prescribed mean curvature equation over general domains, Differential Geom. Appl. 27 (2009), no. 3, 335-343.

[2] T. Bourni, $C^{1, \alpha}$ theory for the prescribed mean curvature equation with Dirichlet data, J. Geom. Anal. 21 (2011), no. 4, 982-1035.

[3] P. Concus and R. Finn, On capillary free surfaces in the absence of gravity, Acta Math. 132 (1974), 177-198.

[4] R. Courant, Dirichlet's Principle, Conformal Mapping, and Minimal Surfaces, Interscience Publishers, New York, N.Y., 1950.

[5] A. K. Echart and K. E. Lancaster, On cusp solutions to a prescribed mean curvature equation, Pacific J. Math. 288 (2017), no. 1, 47-54.

[6] A. R. Elcrat and K. E. Lancaster, On the behavior of a nonparametric minimal surface in a nonconvex quadrilateral, Arch. Rational Mech. Anal. 94 (1986), no. 3, 209-226.

[7] _ Boundary behavior of a nonparametric surface of prescribed mean curvature near a reentrant corner, Trans. Amer. Math. Soc. 297 (1986), no. 2, 645-650.

[8] _ Bernstein functions and the Dirichlet problem, SIAM J. Math. Anal. 20 (1989), no. 5, 1055-1068.

[9] M. Entekhabi and K. Lancaster, Radial limits of bounded nonparametric prescribed mean curvature surfaces, Pacific J. Math. 283 (2016), no. 2, 341-351.

[10] _ Radial limits of capillary surfaces at corners, Pacific J. Math. 288 (2017), no. $1,55-67$.

[11] Boundary continuity of nonparametric prescribed mean curvature surfaces, Taiwanese J. Math. 24 (2020), no. 2, 483-499.

[12] R. Finn, Equilibrium Capillary Surfaces, Grundlehren der Mathematischen Wissenschaften 284, Springer-Verlag, New York, 1986.

[13] _ Moon surfaces, and boundary behaviour of capillary surfaces for perfect wetting and non-wetting, Proc. London Math. Soc. s3-57 (1988), no. 3, 542-576. 
[14] D. Gilbarg and N. S. Trudinger, Elliptic Partial Differential Equations of Second Order (revised second edition), Grundlehren der Mathematischen Wissenschaften 224, Springer-Verlag, New York, 1998.

[15] E. Giusti, On the equation of surfaces of prescribed mean curvature: Existence and uniqueness without boundary conditions, Invent. Math. 46 (1978), no. 2, 111-137.

[16] K. Hayasida and M. Nakatani, On the Dirichlet problem of prescribed mean curvature equations without H-convexity condition, Nagoya Math. J. 157 (2000), 177-209.

[17] A. Korn, Über Minimalflächen, deren Randkurven wenig von ebenen Kurven abweichen, Abh. Königl. Preuss. Akad. Wiss., Berlin, Phys.-Math. Cl. II (1909), 1-37.

[18] K. E. Lancaster, Boundary behavior of a nonparametric minimal surface in $\mathbb{R}^{3}$ at a nonconvex point, Analysis 5 (1985), no. 1-2, 61-69.

[19] Nonparametric minimal surfaces in $\mathbb{R}^{3}$ whose boundaries have a jump discontinuity, Internat. J. Math. Math. Sci. 11 (1988), no. 4, 651-656.

[20] _ Existence and nonexistence of radial limits of minimal surfaces, Proc. Amer. Math. Soc. 106 (1989), no. 3, 757-762.

[21] K. E. Lancaster and D. Siegel, Existence and behavior of the radial limits of a bounded capillary surface at a corner, Pacific J. Math. 176 (1996), no. 1, 165-194. Correction to "Existence and behavior of the radial limits of a bounded capillary surface at a corner", Pacific J. Math. 179 (1997), no. 2 , 397-402.

[22] C. P. Lau, Quasilinear elliptic equations with small boundary data, Manuscripta Math. 53 (1985), no. 1-2, 77-99.

[23] F.-H. Lin, Behaviour of nonparametric solutions and free boundary regularity, in: Miniconference on Geometry and Partial Differential Equations 2 (Canberra, 1986), 96-116, Proc. Centre Math. Anal. Austral. Nat. Univ. 12, Austral. Nat. Univ., Canberra, 1987.

[24] J. C. C. Nitsche, Lectures on Minimal Surfaces, Vol. 1: Introduction, fundamentals, geometry and basic boundary value problems, Cambridge University Press, Cambridge, 1989.

[25] J. Ripoll and F. Tomi, On solutions to the exterior Dirichlet problem for the minimal surface equation with catenoidal ends, Adv. Calc. Var. 7 (2014), no. 2, 205-226. 
[26] J. Serrin, The problem of Dirichlet for quasilinear elliptic differential equations with many independent variables, Philos. Trans. Roy. Soc. London Ser. A 264 (1969), $413-496$.

[27] G. H. Williams, The Dirichlet problem for the minimal surface equation with Lipschitz continuous boundary data, J. Reine Angew. Math. 354 (1984), 123-140.

[28] _ The Dirichlet problem for the minimal surface equation, in: Miniconference on Nonlinear Analysis (Canberra, 1984), 233-239, Proc. Centre Math. Appl. Austral. Nat. Univ. 8, Austral. Nat. Univ., Canberra, 1984.

Mozhgan Nora Entekhabi

Department of Mathematics, Florida A \& M University, Tallahassee, FL 32307, USA

E-mail address: mozhgan.entekhabi@famu.edu

Kirk Eugene Lancaster

Wichita, Kansas 67226, USA

E-mail address: redwoodsrunner@gmail.com 